\title{
Supporting Interaction in Public Space with Electrical Muscle Stimulation
}

Max Pfeiffer

Human-Computer Interaction Group

University of Hannover

max.pfeiffer@hci.uni-hannover.de

Stefan Schneegass, Florian Alt

Institute for Visualization and Interactive Systems

University of Stuttgart

\{firstname.lastname $\}$ Qvis.uni-stuttgart.de

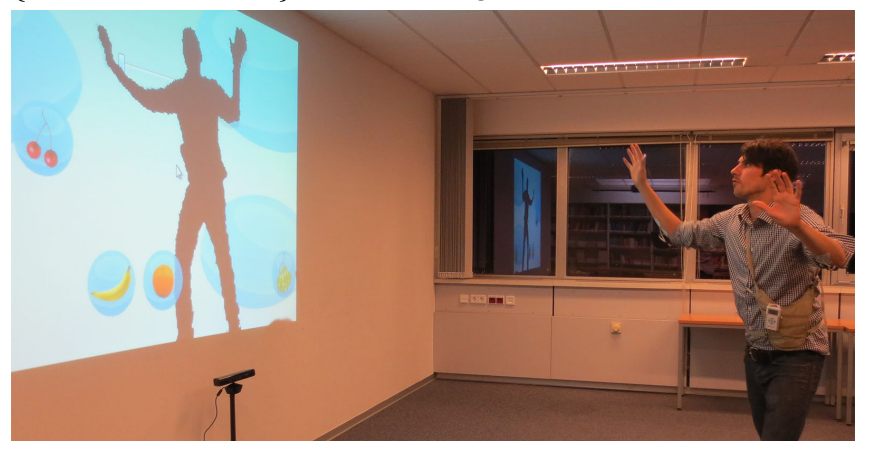

Figure 1: A user is interacting with the system perceiving EMS feedback.

Permission to make digital or hard copies of part or all of this work for personal or classroom use is granted without fee provided that copies are not made or distributed for profit or commercial advantage and that copies bear this notice and the full citation on the first page. Copyrights for third-party components UbiComp'13 Adjunct, September 8-12, 2013, Zuich, Switzerland: ACM 978-1-4503-2215-7/13/09.

\begin{abstract}
As displays in public space are augmented with sensors, such as the Kinect, they enable passersby to interact with the content on the screen. As of today, feedback on the user action in such environments is usually limited to the visual channel. However, we believe that more immediate and intense forms, in particular haptic feedback, do not only increase the user experience, but may also have a strong impact on user attention and memorization of the content encountered during the interaction. Haptic feedback can today be achieved through vibration on the mobile phone, which is strongly dependent on the location of the device. We envision that fabrics, such as underwear, can in the future be equipped with electrical muscle stimulation, thus providing a more natural and direct way of haptic feedback. In this demo we aim to showcase the potential of applying electrical muscle stimulation as direct haptic feedback during interaction in public spaces in the context of a Kinect-based game for public displays.
\end{abstract}

\section{Author Keywords}

interactivity, tactile feedback, EMS, public spaces

\section{ACM Classification Keywords}

H.5.2 [Information interfaces and presentation]: User Interfaces - evaluation/methodology, haptic $\mathrm{I} / \mathrm{O}$, input devices and strategies, interaction styles. 


\section{Introduction}

Providing feedback is one of the Golden Rules of user interface design as proposed by Shneiderman and Plaisant [8]. Desktop computers, for example provide pop-up boxes or present feedback in the form of sound effects. Currently, interfaces deployed in public spaces adopt such feedback techniques, for example through providing visual cues on the screen or through acoustic signals [2].This kind of feedback, however, is often inappropriate or insufficient in public spaces. Due to multiple users in front of the display and large interaction distances, visual cues may be obstructed and go unnoticed with the viewer and acoustic signals may disturb passersby or remain unheard. In contrast, users of mobile devices often prefer haptic feedback, which is more subtle, privacy preserving, cannot be obstructed, and does not disturb others. Therefore, we investigate using haptic feedback for interactive installations in public spaces.

In the future, haptic feedback may be generated in different ways, including force feedback. The technology for such feedback methods is getting smaller and is integrated nowadays in mobile devices [6]. We imagine that the feedback can be provided through bracelets or smart clothing as well. The environment could, through a suitable communication infrastructure, communicate with such feedback devices and thus augment interaction in public space. As a result of this we expect an impact on the users' opinion, experience, and cognition of interactive public applications, which we aim to explore in our work.

With this demonstrator, we want to showcase the potential of haptic feedback with interactive display applications. We show an interactive game that generates haptic feedback utilizing an electrical muscle stimulation (EMS) system as the user plays in front of the screen.

\section{Background and Related Work}

Public displays usually provide visual or acoustic

feedback [2]. In contrast, mobile phones as well as game controllers (e.g., Wii) use haptic feedback acknowledging the execution of a command. This feedback has several advantages such as directness, it preserves the user's privacy, and it can be easily mapped to user interaction.

One way to provide haptic feedback is to use small electric pulses applied to the user's muscles. Electricity is being used to manipulate human bodies since the 18th century [3]. Strojnik et al. [9] used electrical stimulation of muscles in rehabilitation for practicing walking in the late 70th. Recently, electrical muscle stimulation (EMS) was used as force feedback in video game controllers. Kruijff et al. [5] use EMS for pseudo feedback in 3D computer games. In a study they showed that users do not feel pain and rank the feedback as neutral. In [4], Farbiz et al. present an EMS feedback system for mixed reality. The system visualizes a virtual ball that can be hit by a physical racket and gives the expected feedback. In PossessedHand an EMS-based system for controlling finger movements is presented [10]. They show how feedback on single fingers can be used for virtual objects, navigation, and to practice playing instruments. Lopes and Baudisch [6] investigate a mobile EMS system for force feedback in mobile games. In a study they showed that at maximum stimulation and a duration of $1000 \mathrm{~ms}$ the users could provide an average force of $18.7 \mathrm{~N}$ (1903 g).

Prior work shows that the potential of haptic feedback is well understood - particularly for games and in single user environments. We draw upon and extend this knowledge to interaction in public space with the aim to not only tackle the challenges arising in such settings but to also investigate aspects going beyond the feedback itself. 


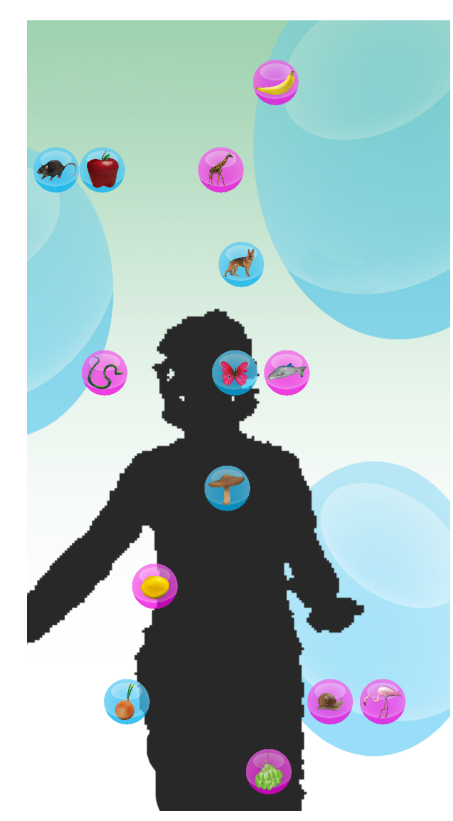

Figure 2: A user is interacting with the soap bubble game.

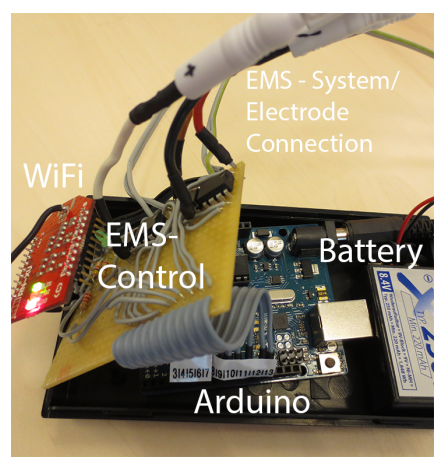

Figure 3: The EMS system generating the haptic feedback.

\section{Demonstrator}

The demonstrator consists of a public display node running a virtual soap bubble game (see Figure 2) and the mobile EMS feedback system that is attached to the user's arms (see Figure 3).

\section{Public Display Node}

In order to study the influence of feedback during the interaction process with a public display, we implemented a soap bubble game [1]. In this game, small soap bubbles containing arbitrary items are floating from the bottom to the top of the screen. Due to the integration of a Microsoft Kinect into the demonstrator, the user is capable of interacting with the game using body movements. We draw upon the user's silhouette to communicate the interactivity of the display [7] and to provide the user feedback on his movement. The silhouette is derived from the depth image of the nearest user in the Kinect's field of view. As soon as the the user's hands touch the soap bubbles they pop and the containing item falls down.

\section{Electrical Muscle Stimulation Feedback}

To provide haptic feedback we use a low-cost, commercial EMS based massage device. As the user touches the bubble the display application sends a signal to the EMS control system which then provides an electrical impulse contracting the muscles of the user as feedback. The EMS system produces a neuromuscular electrical stimulation impulse with a pulse duration of $260 \mu \mathrm{s}$. and pulse frequency from 50 to $70 \mathrm{~Hz}$.

We use an Arduino for controlling the intensity and duration of the feedback. Communication between the Arduino and the display node is established using WiFi. Both lower arms of the user are connected to the system by two electrodes. As soon as the user hits a soap bubble, this arm receives the feedback with a duration of $750 \mathrm{~ms}$. Due to the fact that each user perceives EMS slightly different, the user needs to calibrate the level of intensity before using the system. Since the EMS system and the Arduino are battery-supplied the system is mobile and can be used on the go with no cable hampering the interaction.

\section{Deployment}

We deployed the prototype on an interactive display in our lab to gather early feedback from users. We recruited passersby on an opportunity basis and let them try out the system by playing the game.

Short semi-structured interviews revealed that users could easily map the popping of the soap bubbles to the haptic feedback. One participant reported that he could even feel the difference between one and two simultaneously popping bubbles. Some participants could image to use the system to feel virtual objects shown on the screen as if they were physically touching it. Another user reported that after playing the game for a while he would even miss the feedback if the system failed to trigger the stimulation.

Overall feedback was very positive. However, participants also expressed their skepticism due to the use of electricity, which should be further explored in future work.

\section{Discussion and Conclusion}

We presented a system for providing haptic feedback in the context of an interactive public display application using EMS. EMS technology offers a wide variety of haptic feedback, ranging from a small tickle to large muscle contraction. This allows the impact of different intensities of feedback to be investigated in depth. With the rise of smart and interactive clothes, this kind of 
feedback may be commonly used to provide additional, privacy preserving feedback to the user.

Despite the fact that this system has a lot of potential, some challenges arise with this new kind of technology.

First, the amount of electricity generated needs to be adapted to each user. Applying too strong currents results in pain whereas too little results in no effect at all. As feedback devices will be user-owned we envision an initial calibration that can be used for subsequent use thus limiting the burden for the user. Second, accurately mapping the feedback to the interaction is crucial as delays may lead to users to correctly associating it with their action and thus having a negative impact on the envisioned effect.

Early user feedback shows that by carefully addressing these challenges, a pleasant UX could be provided that allows further research questions to be addressed.

\section{Future Work}

In the future, we are interested in investigating the effects such haptic feedback may have on the user as they interact in public space. We hypothesize an increase in reaction times, a positive effect on user cognition with regard to the content, and an influence on the user experience. The aforementioned effects may strongly depend on a number of different aspects, including but not limited to the duration, intensity, or pattern of the applied feedback as well as the location of the actuator on the body. With the demonstrator we aim to identify and investigate further aspects and gather early user feedback.

Furthermore, we want to explore if EMS feedback can be used to increase the attractiveness of interactive public spaces and whether it can be used to communicate their interactivity.

\section{Acknowledgements}

The research leading to these results has received funding from the European Union 7th Framework Programme ([FP7/2007-2013]) under grant agreement no. 244011 and 600851 .

\section{References}

[1] Alt, F., Schneegass, S., and Schmidt, A. Cognitive effects of interactive public display applications. In Proc. of PerDis '13, ACM (New York, NY, USA, 2013), 6.

[2] Alt, F., Schneegass, S., Schmidt, A., Müller, J., and Memarovic, N. How to evaluate public displays. In Proc. of PerDis '12, ACM (New York, NY, USA, 2012).

[3] Elsenaar, A., and Scha, R. Electric Body Manipulation as Performance Art: A Historical Perspective. Leonardo Music Journal 12 (Dec. 2002), 17-28.

[4] Farbiz, F., Yu, Z. H., Manders, C., and Ahmad, W. An electrical muscle stimulation haptic feedback for mixed reality tennis game. In ACM SIGGRAPH 2007 posters, ACM (New York, NY, USA, 2007).

[5] Kruijff, E., Schmalstieg, D., and Beckhaus, S. Using neuromuscular electrical stimulation for pseudo-haptic feedback. Proc. of VRST '06 (2006), 316.

[6] Lopes, P., and Baudisch, P. Muscle-propelled force feedback: bringing force feedback to mobile devices. In Proc. of CHI'13, ACM (New York, NY, USA, 2013).

[7] Müller, J., Walter, R., Bailly, G., Nischt, M., and Alt, F. Looking Glass: A Field Study on Noticing Interactivity of a Shop Window. In Proc. of CHI '12, ACM (New York, NY, USA, 2012), 297-306.

[8] Shneiderman, B., and Plaisant, C. Designing the User Interface (4th Edition). Pearson Addison Wesley, 2004.

[9] Strojnik, P., Kralj, A., and Ursic, I. Programmed sixchannel electrical stimulator for complex stimulation of leg muscles during walking. IEEE Bio. Eng., 2 (1979).

[10] Tamaki, E., Miyaki, T., and Rekimoto, J. Possessedhand: techniques for controlling human hands using electrical muscles stimuli. In Proc. of CHI '11, ACM (New York, NY, USA, 2011), 543-552. 